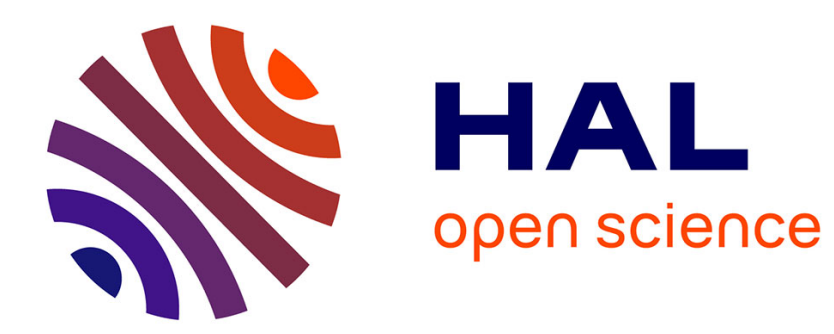

\title{
An interesting family of Black-Scholes perpetuities
}

Bernard Roynette, Marc Yor

\section{To cite this version:}

Bernard Roynette, Marc Yor. An interesting family of Black-Scholes perpetuities. 2008. hal00344713

\section{HAL Id: hal-00344713 \\ https://hal.science/hal-00344713}

Preprint submitted on 5 Dec 2008

HAL is a multi-disciplinary open access archive for the deposit and dissemination of scientific research documents, whether they are published or not. The documents may come from teaching and research institutions in France or abroad, or from public or private research centers.
L'archive ouverte pluridisciplinaire HAL, est destinée au dépôt et à la diffusion de documents scientifiques de niveau recherche, publiés ou non, émanant des établissements d'enseignement et de recherche français ou étrangers, des laboratoires publics ou privés. 


\title{
An interesting family of Black-Scholes perpetuities
}

\author{
B. Roynette ${ }^{(1)}$, M. Yor ${ }^{(2),(3)}$
}

$12 / 11 / 2008$

(1) Institut Elie Cartan, Université Henri Poincaré,

B.P. 239, 54506 Vandoeuvre les Nancy Cedex

email : bernard.roynette@iecn.u-nancy.fr

(2) Laboratoire de Probabilités et Modèles Aléatoires,

Université Paris VI et VII, 4 place Jussieu - Case 188

F - 75252 Paris Cedex 05

email : deaproba@proba.jussieu.fr

(3) Institut Universitaire de France

Abstract We obtain the Laplace transform and integrability properties of the integral over $\mathbb{R}_{+}$of the call quantity associated with geometric Brownian motion with negative drift, thus adding a new element to the list of already studied Brownian perpetuities.

Key words Bessel-McDonald functions, Sturm-Liouville equation, perpetuities.

AMS Classification $60 \mathrm{~J} 25,60 \mathrm{~J} 65,60 \mathrm{G} 44$.

\section{Motivation, introduction}

1.1 Let $\left(B_{t}, t \geq 0\right)$ denote 1-dimensional Brownian motion starting from 0 ; associated to $B$, one considers the geometric Brownian motion :

$$
\left(\mathcal{E}_{t}:=\exp \left(B_{t}-\frac{t}{2}\right), t \geq 0\right)
$$

which is a positive martingale converging a.s. to 0 as $t \rightarrow \infty$. In recent years, the following questions have been asked to the second author, in connection with European option pricing :

i) to express as simply as possible the quantity :

$$
\int_{0}^{\infty} \lambda e^{-\lambda t} E\left(\left(\mathcal{E}_{t}-k\right)^{+}\right) d t \quad(k \geq 0)
$$

ii) to find the law of $\int_{0}^{s}\left(\mathcal{E}_{t}-k\right)^{+} d t$, for fixed $s$

iii) to find the law of $\int_{0}^{\infty} \lambda e^{-\lambda t}\left(\mathcal{E}_{t}-k\right)^{+} d t$ 
i) may be solved quite explicitly, and indeed this has led the authors, jointly with D. Madan, to write a series of papers relating European option prices and laws of last passage times of continuous positive martingales converging to 0 as $t \rightarrow \infty$ (see, e.g. [MRY1], [MRY2], [MRY3]).

Questions ii) and iii) are harder to solve explicitly, as, indeed, one may look for double Laplace transforms of either quantities, e.g. :

$$
\int_{0}^{\infty} e^{-\mu t} E\left(e^{-\lambda \int_{0}^{t}\left(\mathcal{E}_{s}-k\right)^{+} d s}\right) d t
$$

In the present paper, we are studying thoroughly the law of $\int_{0}^{\infty}\left(\mathcal{E}_{t}-k\right)^{+} d t$ which seems to be slightly less difficult than either question ii) or iii). However, the results we obtain are not particulary simple.

1.2 These motivations having been presented, we now concentrate exclusively on the law of $\int_{0}^{\infty}\left(\mathcal{E}_{t}-k\right)^{+} d t$. It will be convenient to consider the two-parameter process :

$$
\left(\mathcal{E}_{t}^{(x)}:=x \exp \left(B_{t}-\frac{t}{2}\right)=\exp \left(\log x+B_{t}-\frac{t}{2}\right), t \geq 0\right)
$$

$\left(\mathcal{E}_{t}^{(x)}, t \geq 0, x>0\right)$ is a Markov process taking values in $\mathbb{R}_{+}$which, most often, we shall denote as : $\left(\left(\mathcal{E}_{t}, t \geq 0 ; P_{x}, x>0\right)\right.$ since $\mathcal{E}_{0}^{(x)}=x$.

To simplify notation, we shall write $P$ for $P_{1}$ and $\mathcal{E}_{t}$ for $\mathcal{E}_{t}^{(1)}$.

For any $k>0$, let us define :

$$
\Pi_{k}^{(x)}:=\int_{0}^{\infty}\left(\mathcal{E}_{t}^{(x)}-k\right)^{+} d t
$$

Again, to simplify, we shall write $\Pi_{k}$ for $\Pi_{k}^{(1)}$. Since $\mathcal{E}_{t}^{(x)} \underset{t \rightarrow \infty}{\longrightarrow} 0$ a.s., the integral which defines (1.2) is a.s. convergent, as $\left(\mathcal{E}_{t}^{(x)}-k\right)^{+}=0$ for $t \geq \mathcal{G}_{k}:=\sup \left\{t ; \mathcal{E}_{t}^{(x)}=k\right\}$, and $\mathcal{G}_{k}<\infty$ a.s.

1.3 We now explain about some reductions of the study of the laws of $\Pi_{k}^{(x)}$, to closely related problems.

i) From Itô's formula, we deduce :

$$
\mathcal{E}_{t}^{(x)}=x+\int_{0}^{t} \mathcal{E}_{s}^{(x)} d B_{s}=\beta_{A_{t}^{(x)}}
$$

where $\left(\beta_{u}, u \geq 0\right)$ denotes the Dubins-Schwarz Brownian motion associated with $\left(\mathcal{E}_{t}^{(x)}, t \geq 0\right)$ (and $\beta_{0}=x$ ) and :

$$
A_{t}^{(x)}=<\mathcal{E}^{(x)}>_{t}=\int_{0}^{t}\left(\mathcal{E}_{s}^{(x)}\right)^{2} d s
$$


Hence :

$$
\Pi_{k}^{(x)}=\int_{0}^{\infty}\left(\beta_{A_{s}^{(x)}}-k\right)^{+} d s=\int_{0}^{T_{0}(\beta)} \frac{\left(\beta_{v}-k\right)^{+}}{\beta_{v}^{2}} d v
$$

(after the change of variable $A_{s}^{(x)}=v$ and where $T_{0}(\beta)=\inf \left\{u \geq 0 ; \beta_{u}=0\right\}$ )

$$
=\int_{k}^{\infty} \frac{(y-k)}{y^{2}} L_{T_{0}(\beta)}^{y} d y \quad\left(=\int_{k}^{\infty} d z \int_{z}^{\infty} \frac{d y}{y^{2}} L_{T_{0}(\beta)}^{y}\right)
$$

from the density of occupation formula, and where $L_{T_{0}}^{y}$ denotes the local time at time $T_{0}$ and level $y$ of Brownian motion $\left(\beta_{u}, u \geq 0\right)$.

ii) When $x=k$, the first Ray-Knight Theorem allows us to write, from (1.6) :

$$
\Pi_{k}^{(k)}=\int_{k}^{\infty} \frac{(y-k)}{y^{2}} \lambda_{y-k} d y
$$

where $\left(\lambda_{z}, z \geq 0\right)$, conditionally on $\lambda_{0}=l$, is a 0 -dimensional squared Bessel process starting at $l$, and where $\lambda_{0}$ is an exponential variable with parameter $\frac{1}{2 k}$, i.e. with expectation $2 k$.

1.4 From the elementary relations :

$$
\left.\left(x e^{B_{t}-\frac{t}{2}}-k\right)^{+}=x\left(e^{B_{t}-\frac{t}{2}}-\frac{k}{x}\right)^{+}=k\left(\frac{x}{k}\left(e^{B_{t}-\frac{t}{2}}\right)-1\right)\right)^{+}
$$

valid for every $x, k>0$, we deduce that the law of $\Pi_{k}$ under $P_{x}$ is that of $x \Pi_{\frac{k}{x}}$ under $P$, or that of $k \Pi_{1}$ under $P_{\frac{x}{k}}$. In other words, for every Borel positive function $\varphi$, we have :

$$
E_{x}\left[\varphi\left(\Pi_{k}\right)\right]=E\left[\varphi\left(x \Pi_{\frac{k}{x}}\right)\right]=E_{\frac{x}{k}}\left(\varphi\left(k \Pi_{1}\right)\right)
$$

These relations allow us to reduce our study of the law of $\Pi_{k}$ under $P_{x}$ to that of $\Pi_{1}$ under $P_{\frac{x}{k}}$. We might as well limit ourselves to the study of $\Pi_{k}$ under $P$.

1.5 It is proven in Dufresne [Duf] and Yor [Y] (see also P. Salminen and M. Yor [SY1]) that, for every $a \neq 0$ and $\nu>0$ :

$$
\int_{0}^{\infty} \exp \left(a B_{t}-\nu t\right) d t \stackrel{\text { (law) }}{=} \frac{2}{a^{2} \gamma_{\frac{2 \nu}{a^{2}}}}
$$

where $\gamma_{b}$ denotes a gamma variable with parameter $b$, i.e. :

$$
P\left(\gamma_{b} \in d t\right)=\frac{1}{\Gamma(b)} e^{-t} t^{b-1} d t \quad(t \geq 0)
$$


In particular, for $a=1$ and $\nu=\frac{1}{2}$

$$
\Pi_{0} \stackrel{(\text { law })}{=} \frac{2}{\gamma_{1}}=\frac{2}{\mathfrak{e}}
$$

where $\mathfrak{e}$ is a standard exponential variable.

1.6 Let $\nu \neq 0$ and :

$$
\mathcal{E}_{t}^{(x, \nu)}:=\exp \left(\nu\left(\log x+B_{t}\right)-\frac{\nu^{2} t}{2}\right)=x^{\nu} \exp \left(\nu B_{t}-\frac{\nu^{2} t}{2}\right)
$$

and define, for $k>0$ :

$$
\Pi_{k}^{(x, \nu)}:=\int_{0}^{\infty}\left(\mathcal{E}_{s}^{(x, \nu)}-k\right)^{+} d s
$$

Since, by scaling, $\left(\mathcal{E}_{t}^{(x, \nu)}, t \geq 0\right) \stackrel{(\text { law })}{=}\left(x^{\nu} \mathcal{E}_{\nu^{2} t}, t \geq 0\right)$, we have :

$$
\begin{aligned}
\Pi_{k}^{(x, \nu)} & \stackrel{\stackrel{\text { law })}{=}}{=} \int_{0}^{\infty}\left(x^{\nu} \mathcal{E}_{\nu^{2} s}-k\right)^{+} d s=\frac{x^{\nu}}{\nu^{2}} \int_{0}^{\infty}\left(\mathcal{E}_{v}-\frac{k}{x^{\nu}}\right)^{+} d v \\
& \stackrel{(\text { law })}{=} \frac{x^{\nu}}{\nu^{2}} \Pi_{\frac{k}{x^{\nu}}}
\end{aligned}
$$

Thus, the study of the law of $\Pi_{k}^{(x, \nu)}$ may be reduced very simply to that of $\Pi_{\frac{k}{x^{\nu}}}$. This is the reason why we have chosen, in this paper, to limit ourselves to $\nu=1$.

\subsection{Here are our results :}

Theorem 1.1 Let $\alpha \geq 0$. Then, for every $x>0, E_{x}\left(\left(\Pi_{1}\right)^{\alpha}\right)<\infty$ if and only if $\alpha<1$

Theorem 1.2 Let $\alpha<0$ and $x>0$. Then:

i) For every $x>1, E_{x}\left(\left(\Pi_{1}\right)^{\alpha}\right)<\infty$.

ii) For every $x<1, P_{x}\left(\Pi_{1}=0\right)=1-x$; hence $E_{x}\left(\left(\Pi_{1}\right)^{\alpha}\right)=+\infty$.

iii) For $x=1, E_{1}\left(\left(\Pi_{1}\right)^{\alpha}\right)<\infty$ if and only if $|\alpha|<\frac{1}{3}$.

Theorem 1.3 (Laplace transform of $\Pi_{1}$ ). For every $\theta \geq 0$ :

$$
E_{x}\left(e^{-\frac{\theta}{2} \Pi_{1}}\right)=\left\{\begin{array}{cl}
\frac{\sqrt{x} K_{\gamma}(\sqrt{4 \theta x})}{\frac{1}{2} K_{\gamma}(\sqrt{4 \theta})-\sqrt{\theta} K_{\gamma}^{\prime}(\sqrt{4 \theta})} & \text { if } x \geq 1 \\
1+x \frac{\frac{1}{2} K_{\gamma}(\sqrt{4 \theta})+\sqrt{\theta} K_{\gamma}^{\prime}(\sqrt{4 \theta})}{\frac{1}{2} K_{\gamma}(\sqrt{4 \theta})-\sqrt{\theta} K_{\gamma}^{\prime}(\sqrt{4 \theta})} & \text { if } 0<x \leq 1
\end{array}\right.
$$


where $K_{\gamma}$ denotes the Bessel-McDonald function with index $\gamma$ (see [Leb], p. 108) and where $\gamma=\sqrt{1-4 \theta}$ if $4 \theta \leq 1$ and $\gamma=i \sqrt{4 \theta-1}$ if $4 \theta \geq 1$.

We shall also prove, as a consequence of Theorem 1.3 :

Theorem 1.4 Let $\left(\lambda_{x}, x \geq 0\right)$ denote a squared Bessel process with dimension 0 started at $l$ and denote its law by $Q_{l}^{(0)}$. Then:

- If $4 \theta \geq 1$ :

$$
\begin{aligned}
Q_{l}^{(0)} & \left(\exp -\frac{\theta}{2} \int_{1}^{\infty} \frac{(x-1)}{x^{2}} \lambda_{x-1} d x\right)\left(=Q_{l}^{(0)}\left(\exp -\frac{\theta}{2} \int_{0}^{\infty} \frac{x}{(x+1)^{2}} \lambda_{x} d x\right)\right. \\
& =\exp \frac{l}{2}\left(\frac{1}{2}+\frac{\sqrt{\theta} K_{i \nu}^{\prime}(\sqrt{4 \theta})}{K_{i \nu}(\sqrt{4 \theta})}\right)
\end{aligned}
$$

with $\nu=\sqrt{4 \theta-1}$

- If $4 \theta \leq 1$ :

$$
Q_{l}^{(0)}\left(\exp -\frac{\theta}{2} \int_{1}^{\infty} \frac{x-1}{x^{2}} \lambda_{x-1} d x\right)=\exp \frac{l}{2}\left(\frac{1}{2}+\frac{\sqrt{\theta} K_{\nu}^{\prime}(\sqrt{4 \theta})}{K_{\nu}(\sqrt{4 \theta})}\right)
$$

with $\nu=\sqrt{1-4 \theta}$.

In Section 3 of this work, we shall study the asymptotic behavior, as $\theta \rightarrow \infty$, of $E\left(e^{-\frac{\theta}{2} \Pi_{1}}\right)$ and we shall obtain :

$$
E\left(e^{-\frac{\theta}{2} \Pi_{1}}\right) \underset{\theta \rightarrow \infty}{\sim} \frac{C}{\theta^{\frac{1}{3}}} \quad(C>0)
$$

Finally, in a short Section 4, we shall indicate how Theorems 1.1, 1.2 and 1.3 extend when we replace $\Pi_{k}$ by $\Pi_{k}^{(\rho)}$, with :

$$
\begin{aligned}
\Pi_{k}^{(\rho)} & :=\int_{0}^{\infty}\left(e^{\rho\left(B_{t}-\frac{t}{2}\right)}-k\right)^{+} d t \quad(\rho, k>0) \\
& \stackrel{\text { (law) }}{=} \frac{1}{\rho^{2}} \int_{0}^{\infty}\left(e^{\left(B_{u}-\frac{u}{2 \rho}\right)}-k\right)^{+} d u \quad \text { (by scaling) }
\end{aligned}
$$

which, from (1.18), corresponds to consider an extension of our previous perpetuities relative to $\left(B_{t}-\frac{t}{2}, t \geq 0\right)$ to Brownian motion with drift $-\frac{1}{2 \rho}$, i.e. $\left(B_{t}-\frac{t}{2 \rho}, t \geq 0\right)$.

1.8 For studies of other perpetuities related to Brownian motion with drift, we refer the reader to Salminen-Yor ([SY1], [SY2], [SY3]). 


\section{Proofs of Theorems 1.1, 1.2 and 1.3}

\subsection{A first proof of Theorem 1.1}

2.1 i) We now prove that, for every $x>0, E_{x}\left(\Pi_{1}\right)=\infty$ Indeed, from (1.6) :

$$
\begin{aligned}
E_{1}\left(\Pi_{1}\right) & =\int_{1}^{\infty} \frac{(y-1)}{y^{2}} E_{1}\left(L_{T_{0}}^{y}\right) d y \\
& =\int_{1}^{\infty} \frac{(y-1)}{y^{2}} E_{1}\left(L_{T_{0}}^{1}\right) d y \\
& =2 \int_{1}^{\infty} \frac{(y-1)}{y^{2}} d y=+\infty
\end{aligned}
$$

(since, for $y \geq 1, E_{1}\left(L_{T_{0}}^{y}\right)=E_{1}\left(L_{T_{0}}^{1}\right)=2$, as $L_{T_{0}}^{1}$ is an exponential variable with parameter $\left.\frac{1}{2}\right)$.

2.1 ii) We now prove that, for every $x>0, E_{x}\left(\Pi_{1}\right)=\infty$

- For $x \geq 1$, this is clear since $\Pi_{k}^{(x)}$ is an increasing function of $x$ (and a decreasing function of $k$ ). Hence :

$$
E_{x}\left(\Pi_{1}\right) \geq E_{1}\left(\Pi_{1}\right)=+\infty \quad(\text { from }(2.1))
$$

- For $x<1$, from the Markov property :

$$
\Pi_{1}^{(x) \stackrel{(\text { law })}{=}} 1_{\left\{T_{\log \left(\frac{1}{x}\right)}^{\left(-\frac{1}{2}\right)}<\infty\right\}} \cdot \Pi_{1}
$$

with $T_{\log \left(\frac{1}{x}\right)}^{\left(-\frac{1}{2}\right)}:=\inf \left\{t \geq 0 ; B_{t}-\frac{t}{2}=\log \left(\frac{1}{x}\right)\right\}$, and in (2.2), $\Pi_{1}$ and $T_{\log \left(\frac{1}{x}\right)}^{\left(-\frac{1}{2}\right)}$ are assumed independent. Hence :

$$
E\left(\Pi_{1}^{(x)}\right)=P\left(T_{\log \left(\frac{1}{x}\right)}^{\left(-\frac{1}{2}\right)}<\infty\right) E\left(\Pi_{1}\right)=+\infty
$$

from $(2.1)$ and since $P\left(T_{\log \left(\frac{1}{x}\right)}^{\left(-\frac{1}{2}\right)}<\infty\right)=x$ (see Lemma 2.1 below).

2.1 iii) We prove that, for $0 \leq \alpha<1, E_{x}\left(\left(\Pi_{1}\right)^{\alpha}\right)<\infty$ We have :

$$
\begin{aligned}
E_{x}\left(\left(\Pi_{1}\right)^{\alpha}\right) & =x^{\alpha} E\left(\left(\Pi_{\frac{1}{x}}\right)^{\alpha}\right) \quad(\text { from }(1.9)) \\
& \leq x^{\alpha} E\left(\left(\Pi_{0}\right)^{\alpha}\right)
\end{aligned}
$$

(since $\Pi_{k}$ is a decreasing function of $k$ )

$$
\leq x^{\alpha} \int_{0}^{\infty} \frac{2}{y^{\alpha}} e^{-y} d y<\infty \quad(\text { from }(1.12))
$$




\subsection{Second proof of Theorem 1.1}

It hinges upon :

\section{Lemma 2.1}

Let $G_{a}^{(\nu)}:=\sup \left\{u \geq 0 ; B_{u}+\nu u=a\right\} \quad(=0$ if this set is empty) and $T_{a}^{(\nu)}:=\inf \left\{u \geq 0 ; B_{u}+\nu u=a\right\} \quad(=+\infty$ if this set is empty).

i) If $\nu$ and a have the same sign:

- $E\left(e^{\mu G_{a}^{(\nu)}}\right)<\infty$ if and only if $\mu<\frac{\nu^{2}}{2}$

- for every real $\alpha, \quad E\left(\left(G_{a}^{(\nu)}\right)^{\alpha}\right)<\infty$

ii) If $\nu$ and a have opposite signs :

- $E\left(e^{\mu G_{a}^{(\nu)}}\right)<\infty$ if and only if $\mu<\frac{\nu^{2}}{2}$

- for every real $\alpha>0, E\left(\left(G_{a}^{(\nu)}\right)^{\alpha}\right)<\infty$

- $P\left(T_{a}^{(\nu)}=\infty\right)=P\left(G_{a}^{(\nu)}=0\right)=1-e^{2 \nu a}$

Hence, for $\alpha<0, E\left(\left(G_{a}^{(\nu)}\right)^{\alpha}\right)=+\infty$

The proof of this Lemma is obvious. It hinges on the well-known formulae :

- If $\nu, a>0$ :

$$
P\left(G_{a}^{(\nu)} \in d t\right)=\frac{\nu}{\sqrt{2 \pi t}} \exp \left(-\frac{1}{2 t}(a-\nu t)^{2}\right) d t
$$

- If $\nu, a>0$ :

$$
P\left(G_{a}^{(-\nu)} \in d t\right)=\frac{\nu}{\sqrt{2 \pi t}} \exp \left(-\frac{1}{2 t}(a+\nu t)^{2}\right) d t
$$

- If $\nu$ and $a$ have opposites signs :

$$
P\left(G_{a}^{(\nu)}>0\right)=P\left(T_{a}^{(\nu)}<\infty\right)=e^{2 \nu a}
$$

- $G_{a}^{(\nu)} \stackrel{(\text { law })}{=} G_{(-a)}^{(-\nu)} \stackrel{(\text { law })}{=} \frac{1}{T_{\nu}^{(a)}}$

We now give a second proof of Theorem 1.1.

- We first show that, for $0 \leq \alpha<1, E_{x}\left(\left(\Pi_{1}\right)^{\alpha}\right)<\infty$. From the relation :

$$
\begin{aligned}
\Pi_{1}^{(x)} & =x \int_{0}^{\infty}\left(\mathcal{E}_{t}-\frac{1}{x}\right)^{+} d t=x \int_{0}^{G_{-\log x}^{\left(-\frac{1}{2}\right)}}\left(\mathcal{E}_{t}-\frac{1}{x}\right)^{+} d t \\
& \leq x\left(\sup _{t \geq 0} \mathcal{E}_{t}\right) \cdot G_{-\log x}^{\left(-\frac{1}{2}\right)}
\end{aligned}
$$


we deduce that, for $0 \leq \alpha<1, \alpha p<1$ and $\frac{1}{p}+\frac{1}{q}=1(p, q>1)$ :

$$
\begin{aligned}
E_{x}\left(\left(\Pi_{1}\right)^{\alpha}\right) & \leq x^{\alpha} E\left[\left(\sup _{t \geq 0} \mathcal{E}_{t}\right)^{\alpha}\left(G_{-\log x}^{\left(-\frac{1}{2}\right)}\right)^{\alpha}\right] \\
& \leq x^{\alpha} E\left(\left(\sup _{t \geq 0} \mathcal{E}_{t}\right)^{\alpha p}\right)^{\frac{1}{p}} E\left(\left(G_{(-\log x)}^{\left(-\frac{1}{2}\right)}\right)^{\alpha q}\right)^{\frac{1}{q}} \\
& \leq C x^{\alpha} E\left(\left(\sup _{t \geq 0} \mathcal{E}_{t}\right)^{\alpha p}\right)^{\frac{1}{p}} \quad \text { (from Lemma 2.1) }
\end{aligned}
$$

As $\left(\mathcal{E}_{t}, t \geq 0\right)$ is a positive martingale, starting from 1 , and converging a.s. to 0 as $t \rightarrow \infty$, we get (see [NY] or [RY], Chap. II, Ex. 3.12, p. 73) :

$$
\sup _{s \geq 0} \mathcal{E}_{s} \stackrel{(\text { law) }}{=} \frac{1}{U}
$$

with $U$ uniform on $[0,1]$. Thus :

$$
E\left(\left(\sup _{t \geq 0} \mathcal{E}_{t}\right)^{\alpha p}\right)=\int_{0}^{1} \frac{1}{u^{\alpha p}} d u<\infty
$$

since $\alpha p<1$.

- We then show that $E_{x}\left(\Pi_{1}\right)=\infty$. First of all, it follows from [MRY1] that :

$$
E_{1}\left(\left(\mathcal{E}_{t}-k\right)^{+}\right)=P\left(G_{\log k}^{\left(\frac{1}{2}\right)} \leq t\right)
$$

Hence :

$$
E_{1}\left(\Pi_{1}\right)=\int_{0}^{\infty} P\left(G_{0}^{\left(\frac{1}{2}\right)} \leq t\right) d t=E\left(\int_{G_{0}^{\left(\frac{1}{2}\right)}}^{\infty} d t\right)=+\infty
$$

since $G_{0}^{\left(\frac{1}{2}\right)}<+\infty$ a.s.

Likewise :

$$
\begin{aligned}
E_{x}\left(\Pi_{1}\right) & =x E_{1}\left(\Pi_{\frac{1}{x}}\right) \quad(\text { from }(1.9)) \\
& =x E\left(\int_{G_{\log \frac{1}{x}}^{\left(\frac{1}{2}\right)}}^{\infty} d t\right)=\infty
\end{aligned}
$$

from (2.10) and since $G_{\log \frac{1}{x}}^{\left(\frac{1}{2}\right)}<\infty$ a.s. 


\subsection{Proof of Theorem 1.2}

i) We already prove point $i)$ :

Let $x>1$ and $x^{\prime}$ such that $1<x^{\prime}<x$. It is then obvious that :

$$
\Pi_{1}^{(x)} \geq\left(x^{\prime}-1\right) T_{\log \left(\frac{x^{\prime}}{x}\right)}^{\left(-\frac{1}{2}\right)}
$$

(with $T_{a}^{\left(-\frac{1}{2}\right)}:=\inf \left\{t \geq 0 ; B_{t}-\frac{t}{2}=a\right\}$, since, if $t<T_{\log \frac{x^{\prime}}{x}}^{\left(-\frac{1}{2}\right)}$, then $e^{\log x+B_{t}-\frac{t}{2}}-1 \geq x^{\prime}-1$ ). Hence, with $\gamma>0$ :

$$
\begin{aligned}
& E_{x}\left(\frac{1}{\left(\Pi_{1}\right)^{\gamma}}\right)=\int_{0}^{\infty} P_{x}\left(\frac{1}{\left(\Pi_{1}\right)^{\gamma}} \geq t\right) d t=\int_{0}^{\infty} P_{x}\left(\Pi_{1}<v\right) \frac{\gamma}{v^{1+\gamma}} d v \\
& \leq \int_{0}^{\infty} \frac{\gamma}{v^{1+\gamma}} d v P\left(T_{\log \frac{x^{\prime}}{x}}^{\left(-\frac{1}{2}\right)} \leq \frac{v}{x^{\prime}-1}\right) d v \quad(\text { from }(2.11)) \\
& =\int_{0}^{\infty} \frac{\gamma}{v^{1+\gamma}} d v\left(P\left(G_{\frac{1}{2}}^{\left(\log \left(\frac{x}{x^{\prime}}\right)\right)} \geq \frac{x^{\prime}-1}{v}\right) d v\right. \\
& \text { (since } \quad T_{a}^{(\nu)} \stackrel{(\text { law })}{=} \frac{1}{G_{\nu}^{(a)}} \text { and } \quad G_{\nu}^{(a)} \stackrel{(\text { law })}{=} G_{-\nu}^{(-a)} \text { ) } \\
& =\int_{0}^{\infty} \frac{\gamma}{\left(x^{\prime}-1\right)^{\gamma}} u^{\gamma-1} d u P\left(G_{\frac{1}{2}}^{\left(\log \left(\frac{x}{x^{\prime}}\right)\right)} \geq u\right) d u \\
& =\frac{1}{\left(x^{\prime}-1\right)^{\gamma}} E\left(\left(G_{\frac{1}{2}}^{\left(\log \frac{x}{x^{\prime}}\right)}\right)^{\gamma}\right)<\infty
\end{aligned}
$$

(from point i) of Lemma 2.1).

ii) We now prove point $i$ i) :

It is clear that, for $x<1$ :

$$
\left\{\Pi_{1}^{(x)}=0\right\}=\left\{T_{\log \frac{1}{x}}^{\left(-\frac{1}{2}\right)}=\infty\right\}
$$

Thus, $P\left\{\Pi_{1}^{(x)}=0\right\}=P\left\{T_{\log \frac{1}{x}}^{\left(-\frac{1}{2}\right)}=\infty\right\}=P\left\{G_{\frac{1}{2}}^{(\log x)}=0\right\}=1-x>0$ from Lemma 2.1 and (1.26).

iii) We now prove point iii) :

For this purpose, we write, for $\gamma>0$ :

$$
E_{1}\left(\frac{1}{\left(\Pi_{1}\right)^{\gamma}}\right)=\frac{1}{\Gamma(\gamma)} \int_{0}^{\infty} E\left(e^{-\theta \Pi_{1}}\right) \theta^{\gamma-1} d \theta
$$

and we show, in the next Section 3, that :

$$
E_{1}\left(e^{-\theta \Pi_{1}}\right) \underset{\theta \rightarrow \infty}{\sim} \frac{C}{\theta^{\frac{1}{3}}} \quad(C>0)
$$

Thus, $E\left(\frac{1}{\left(\Pi_{1}\right)^{\gamma}}\right)<\infty$ if and only if $\int_{1}^{\infty} \theta^{\gamma-1-\frac{1}{3}} d \theta<\infty$, that is, if and only if $\gamma<\frac{1}{3}$. 


\subsection{Proof of Theorem 1.3}

\section{a) A useful Lemma}

Lemma 2.2 Let $\theta \geq 0$ and $K_{\gamma}$ the Bessel McDonald function with index $\gamma$, such that $\gamma=\sqrt{1-4 \theta}$ if $\theta \leq \frac{1}{4}$ and $\gamma=i \sqrt{4 \theta-1}$ if $\theta \geq \frac{1}{4}$.

1) Define the function $\varphi_{\theta}: \mathbb{R}_{+} \rightarrow \mathbb{R}$ by:

$$
\varphi_{\theta}(y)=\sqrt{y} K_{\gamma}(\sqrt{4 \theta y}) \quad(y \geq 0)
$$

Then :

i) $\varphi_{\theta}$ is a real valued function which satisfies :

$$
\varphi_{\theta}^{\prime \prime}(y)+\left(-\frac{\theta}{y}+\frac{\theta}{y^{2}}\right) \varphi_{\theta}(y)=0
$$

ii) $\varphi_{\theta}$, restricted to $[1, \infty[$ is positive, convex, bounded and decreasing.

2) We define the function $\widetilde{\varphi}_{\theta}: \mathbb{R}_{+} \rightarrow \mathbb{R}_{+}$by :

$$
\widetilde{\varphi}_{\theta}(y)= \begin{cases}\varphi_{\theta}(y) & \text { if } y \geq 1 \\ \left(\varphi_{\theta}(1)-\varphi_{\theta}^{\prime}(1)\right)+y \varphi_{\theta}^{\prime}(1) & \text { if } 0 \leq y \leq 1\end{cases}
$$

Then $\widetilde{\varphi}_{\theta}$ is a bounded, positive, convex, decreasing function which satisfies :

$$
\widetilde{\varphi}_{\theta}^{\prime \prime}(y)+\left(-\frac{\theta}{y}+\frac{\theta}{y^{2}}\right) 1_{y \geq 1} \cdot \widetilde{\varphi}_{\theta}(y)=0
$$

\section{b) Proof of Lemma 2.2}

i) Relation (2.15) (as well as relation (2.17)) follows from a direct computation, using the equation $K_{\gamma}^{\prime \prime}(z)+\frac{1}{z} K_{\gamma}^{\prime}(z)-\left(1+\frac{\gamma^{2}}{z^{2}}\right) K_{\gamma}(z)=0$ (see [Leb], p. 110) and the fact that $\gamma^{2}=1-4 \theta$ (see Petiau [Pet], p. 306, formula (8), which needs to be corrected by replacing $a$ by $-a$, or Kamke [Kam], p. 440).

We distinguish two cases :

Case $1: 4 \theta \leq 1, \gamma=\sqrt{1-4 \theta}$. The function $K_{\gamma}$, hence also $\varphi_{\theta}$, is positive. Furthermore $\varphi_{\theta}$ is bounded on $\mathbb{R}_{+}$since :

$$
\varphi_{\theta}(y) \underset{y \rightarrow 0}{\sim} C y^{\frac{1-\gamma}{2}}, \varphi_{\theta}(y) \underset{y \rightarrow \infty}{\sim} C^{\prime} y^{\frac{1}{4}} e^{-\sqrt{4 \theta y}} \quad \text { (see [Leb], p. } 123 \text { and 136) }
$$

On the other hand, from (2.15), the function $\varphi_{\theta}$ is convex on the interval $[1, \infty[$. As it is convex, positive, and bounded, it is decreasing. Lemma 2.2 is thus proven in this case. 


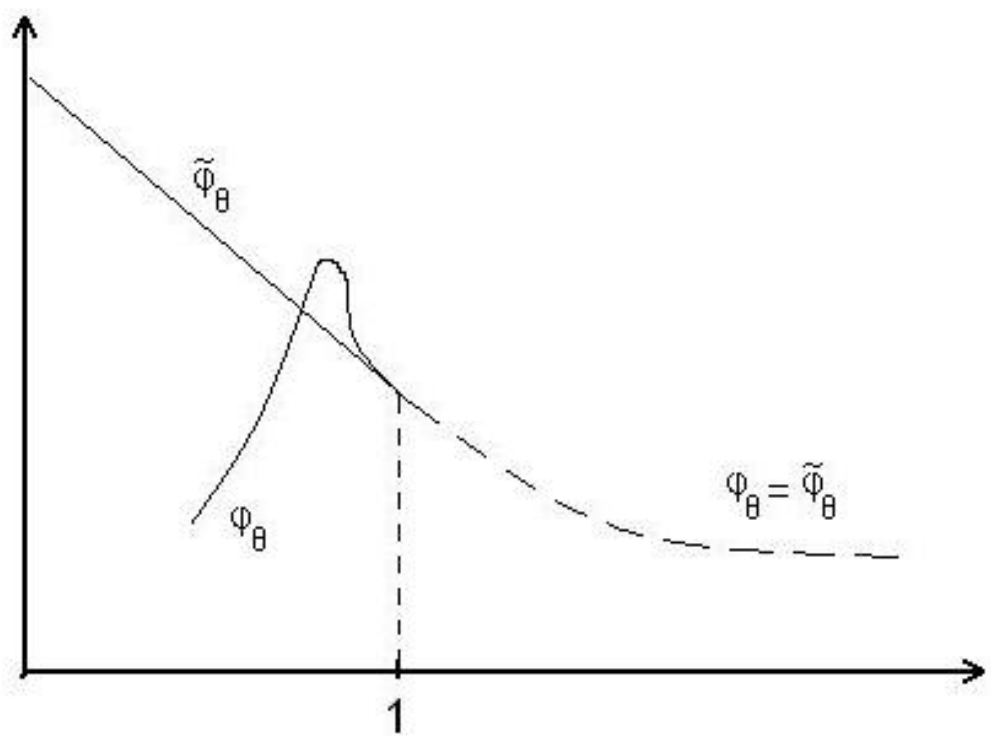

Figure 1: Graphs of $\varphi_{\theta}$ and $\widetilde{\varphi}_{\theta}$

Case $2: 4 \theta \geq 1, \gamma=i \sqrt{4 \theta-1}$ and here $\varphi_{\theta}(y)=\sqrt{y} K_{i \nu}(\sqrt{4 \theta y})$ with $\nu=\sqrt{4 \theta-1}$. From the integral representation formula (see [Leb], p. 119) :

$$
K_{i \nu}(y)=\int_{0}^{\infty} e^{-y \cosh u} \cos (\nu u) d u \quad(y \geq 0)
$$

we deduce that $K_{i \nu}(y)$ is real valued, hence so is $\varphi_{\theta}(y)$.

On the other hand, $\varphi_{\theta}$ is bounded on [0, $\infty$ [. Indeed, for $y \geq 0$, from (2.18) :

$$
\left|\sqrt{4 \theta} \varphi_{\theta}\left(\frac{y^{2}}{4 \theta}\right)\right|=y\left|K_{i \nu}(y)\right| \leq y \int_{0}^{\infty} e^{-y \frac{e^{u}}{2}} d u \leq y \int_{\frac{y}{2}}^{\infty} e^{-v} \frac{d v}{v}
$$

Thus :

$$
\begin{aligned}
& \left|\sqrt{4 \theta} \varphi_{\theta}\left(\frac{y^{2}}{4 \theta}\right)\right| \leq e^{-\frac{y}{4}} \quad \text { for } y \text { large enough, hence } \\
& \left|\sqrt{4 \theta} \varphi_{\theta}\left(\frac{y^{2}}{4 \theta}\right)\right| \underset{y \rightarrow \infty}{\longrightarrow} 0 \quad \text { and } \\
& \left|\sqrt{4 \theta} \varphi_{\theta}\left(\frac{y^{2}}{4 \theta}\right)\right| \leq y(C+|\log (y)|) \underset{y \rightarrow 0}{\longrightarrow} 0
\end{aligned}
$$

On the other hand, it is clear, from $(2.18)$, that $K_{i \nu}(y)>0$ hence $\varphi_{\theta}(y)>0$, for $y$ large enough.

- We now show that $K_{i \nu}$ is decreasing on $\left[\nu, \infty\right.$ [. If not, there would exist a point $y_{0}>\nu$ such that :

$$
K_{i \nu}\left(y_{0}\right)>0, K_{i \nu}^{\prime}\left(y_{0}\right)=0, K_{i \nu}^{\prime \prime}\left(y_{0}\right) \leq 0 .
$$


However,

$$
K_{i \nu}^{\prime \prime}\left(y_{0}\right)+\frac{1}{y_{0}} K_{i \nu}^{\prime}\left(y_{0}\right)=\left(1-\frac{\nu^{2}}{y_{0}^{2}}\right) K_{i \nu}\left(y_{0}\right)
$$

Thus $K_{i \nu}^{\prime \prime}\left(y_{0}\right)>0$, which is absurd. Since $K_{i \nu}$ is decreasing on $[\nu, \infty[$, and positive near $\infty$, $K_{i \nu}$ is positive on $\left[\nu, \infty\right.$ [. Thus, $\varphi_{\theta}$ is positive on [1, $\infty$ [ (since, if $y \geq 1, \sqrt{4 \theta y} \geq \sqrt{4 \theta} \geq$ $\sqrt{4 \theta-1}=\nu)$. From the relation $(2.15)$, we then deduce that $\varphi_{\theta}$ is convex on $[1, \infty[$. Since it is bounded, convex and positive, it is decreasing. Lemma 2.2 is proven.

\section{c) End of the proof of Theorem 1.3.}

Let $\left(M_{t}^{\theta}:=\widetilde{\varphi}_{\theta}\left(B_{t}\right) \exp -\frac{\theta}{2} \int_{0}^{t} \frac{\widetilde{\varphi}_{\theta}^{\prime \prime}}{\varphi_{\theta}}\left(B_{s}\right) d s, t \geq 0\right)$. Then $\left(M_{t}^{\theta}, t \geq 0\right)$ is a local martingale. It is equal, from (2.17) to :

$$
M_{t}^{\theta}=\widetilde{\varphi}_{\theta}\left(B_{t}\right) \exp -\frac{\theta}{2} \int_{0}^{t} \frac{\left(B_{s}-1\right)^{+}}{B_{s}^{2}} d s
$$

and, from Lemma 2.2, for every $x \geq 0,\left(M_{t \wedge T_{0}}^{\theta}, t \geq 0\right)$ is bounded. Thus, from Doob's optional stopping Theorem :

$$
\begin{aligned}
\widetilde{\varphi}_{\theta}(x) & =E_{x}\left(M_{0}^{\theta}\right)=E_{x}\left[\widetilde{\varphi}_{\theta}\left(B_{T_{0}}\right) \exp -\frac{\theta}{2} \int_{0}^{T_{0}(B)} \frac{\left(B_{s}-1\right)^{+}}{B_{s}^{2}} d s\right] \\
& =\widetilde{\varphi}_{\theta}(0) E_{x}\left[\exp -\frac{\theta}{2} \Pi_{1}\right] \quad(\text { from }(1.5))
\end{aligned}
$$

Thus :

$$
E_{x}\left(e^{-\frac{\theta}{2} \Pi_{1}}\right)=\frac{\widetilde{\varphi}_{\theta}(x)}{\widetilde{\varphi}_{\theta}(0)}
$$

This is precisely Theorem 1.3, owing to formula (2.16) which yields $\widetilde{\varphi}_{\theta}$ explicitly.

Remark 2.3 Theorem 1.3 allows to recover formula $(1.9): \Pi_{0} \stackrel{(\text { law) }}{=} \frac{2}{\mathfrak{e}}$. Indeed, on one hand :

$$
E_{1}\left(e^{-\frac{\theta}{2} \Pi_{0}}\right)=E\left(e^{-\frac{\theta}{2} \frac{2}{e}}\right)=\int_{0}^{\infty} e^{-\frac{\theta}{z}-z} d z=2 \sqrt{\theta} K_{1}(\sqrt{4 \theta}) \quad \text { (see [Leb], p. }
$$


and, on the other hand :

$$
\begin{aligned}
& E_{1}\left(e^{-\frac{\theta}{2} \Pi_{0}}\right)=\lim _{\epsilon \downarrow 0} E_{1}\left(e^{-\frac{\theta}{2} \Pi_{\epsilon}}\right)=\lim _{\epsilon \downarrow 0} E\left(\exp -\frac{\theta}{2} \int_{0}^{\infty}\left(e^{B_{t}-\frac{t}{2}}-\epsilon\right)^{+} d t\right) \\
& =\lim _{\epsilon \downarrow 0} E\left(e^{-\frac{\theta \epsilon}{2} \int_{0}^{\infty}\left(\frac{1}{\epsilon}\left(e^{B_{t}-\frac{t}{2}}-1\right)^{+} d t\right.}\right)=\lim _{\epsilon \downarrow 0} E_{\frac{1}{\epsilon}}\left(e^{-\frac{\theta \epsilon}{2} \Pi_{1}}\right) \\
& =\lim _{\epsilon \downarrow 0} \frac{\frac{1}{\sqrt{\epsilon}} K_{\sqrt{1-4 \theta \epsilon}}(\sqrt{4 \theta})}{\frac{1}{2} K_{\sqrt{1-4 \theta \epsilon}}(\sqrt{4 \theta \epsilon})-\frac{1}{2} \sqrt{4 \theta \epsilon} K_{\sqrt{1-4 \theta \epsilon}}^{\prime}(\sqrt{4 \theta \epsilon})} \\
& \text { (from (1.5), replacing } \theta \text { by } \theta \epsilon \text { and } x \text { by } \frac{1}{\epsilon} \text { ) } \\
& =\lim _{\epsilon \downarrow 0} \frac{\frac{1}{\sqrt{\epsilon}} K_{1}(\sqrt{4 \theta})+O(1)}{\frac{1}{2} K_{\sqrt{1-4 \theta \epsilon}}(\sqrt{4 \theta \epsilon})[1-\sqrt{1-4 \theta \epsilon}]+\sqrt{\theta \epsilon} K_{\sqrt{1-4 \theta \epsilon}+1}(\sqrt{4 \theta \epsilon})} \\
& \text { (since } z K_{\nu}^{\prime}(z)=\nu K_{\nu}(z)-z K_{\nu+1}(z) \text {; see [Leb], p. 110) } \\
& =\lim _{\epsilon \downarrow 0} \frac{\frac{1}{\sqrt{\epsilon}} K_{1}(\sqrt{4 \theta})+O(1)}{O(1)+\sqrt{\theta \epsilon}\left(\frac{1}{2 \theta \epsilon}+O(1)\right)}=2 \sqrt{\theta} K_{1}(\sqrt{4 \theta})
\end{aligned}
$$

\subsection{Proof of Theorem 1.4}

From Theorem 1.3, we know that, for $\theta \geq \frac{1}{4}$ :

$$
\begin{aligned}
E_{1}\left(e^{-\frac{\theta}{2} \Pi_{1}}\right) & =\frac{2 K_{i \nu}(\sqrt{4 \theta})}{K_{i \nu}(\sqrt{4 \theta})-\sqrt{4 \theta} K_{i \nu}^{\prime}(\sqrt{4 \theta})} \\
& =\frac{1}{\frac{1}{2}-\sqrt{\theta} \frac{K_{i \nu}^{\prime}(\sqrt{4 \theta})}{K_{i \nu}(\sqrt{4 \theta})}}=\frac{1}{2} \int_{0}^{\infty} e^{-\frac{l}{2}+\frac{l}{2}\left(\frac{1}{2}+\sqrt{\theta} \frac{K_{i \nu}^{\prime}(\sqrt{4 \theta})}{K_{i \nu}(\sqrt{4 \theta})} d l\right.}
\end{aligned}
$$

On the other hand, from (1.7) :

$$
\begin{aligned}
E_{1}\left(e^{-\frac{\theta}{2} \Pi_{1}}\right) & =E_{1}\left(\exp -\frac{\theta}{2} \int_{1}^{\infty} \frac{(y-1)^{+}}{y^{2}} \lambda_{y-1} d y\right) \\
& =\frac{1}{2} \int_{0}^{\infty} e^{-\frac{l}{2}} d l E\left(\exp -\frac{\theta}{2} \int_{1}^{\infty} \frac{(y-1)^{+}}{y^{2}} \lambda_{y-1} d y \mid \lambda_{0}=l\right) \\
& =\frac{1}{2} \int_{0}^{\infty} e^{-\frac{l}{2}} d l Q_{l}^{(0)}\left(\exp -\frac{\theta}{2} \int_{0}^{\infty} \frac{y}{(1+y)^{2}} \lambda_{y} d y\right)
\end{aligned}
$$

where $Q_{l}^{(0)}$ denotes the expectation relative to a squared Bessel process of dimension 0 , starting from $l$. The comparison of (2.21) and (2.20) implies Theorem 1.4 in the case 
$\theta \geq \frac{1}{4}$, since the Laplace transform is one-to-one. The proof of Theorem 1.4 in the case $\theta \leq \frac{1}{4}$ is the same.

Remark 2.4 Let $\mu$ denote a positive $\sigma$-finite measure on $\mathbb{R}_{+}$. Let $\Phi$ denote the unique decreasing, positive solution on $\mathbb{R}_{+}$of the Sturm-Liouville equation $\Phi^{\prime \prime}=\mu \Phi$ (and such that $\Phi(0)=1$ ). It is well known (see [RY], Chap. IX, p. 444) that :

$$
Q_{l}^{(0)}\left(\exp \left(-\frac{1}{2} \int_{0}^{\infty} \lambda_{y} \mu(d y)\right)\right)=\exp \left(\frac{l}{2} \Phi^{\prime}\left(0_{+}\right)\right)
$$

(Observe that, as $\left(\lambda_{y}, y \geq 0\right)$ has compact support a.s., $\int_{0}^{\infty} \lambda_{y} \mu(d y)<\infty$ if $\mu$ is $\sigma$-finite on $\mathbb{R}_{+}$).

Theorem 1.4 may be recovered easily by applying formula $(2.22)$ with $\mu(d y)=\frac{y}{1+y^{2}} d y$. On the other hand, if $\mu(d y)=a(y) d y$, with

$$
c_{1} 1_{\left[0, \gamma_{1}\right]}(y) \leq a(y) \leq c_{2} 1_{\left[0, \gamma_{2}\right]}(y) \quad\left(0<c_{1} \leq c_{2}, 0<\gamma_{1} \leq \gamma_{2}\right),
$$

we deduce from [RY], Chap. XI, Corollary 1.8, that :

$$
\exp -\frac{l}{2} c_{2}^{\prime} \sqrt{\theta} \leq E_{l}^{(0)}\left(e^{-\frac{\theta}{2} \int_{0}^{\infty} \lambda_{y} \mu(d y)}\right) \leq \exp -\frac{l}{2} c_{1}^{\prime} \sqrt{\theta}
$$

for $\theta$ large enough, whereas, as we shall show in Section 3 :

$$
Q_{l}^{(0)}\left(\exp -\frac{\theta}{2} \int_{0}^{\infty} \frac{y}{1+y^{2}} \lambda_{y} d y\right) \underset{\theta \rightarrow \infty}{\sim} C e^{-\frac{l}{2} \theta^{\frac{1}{3}}}
$$

\section{Asymptotic behavior of $E_{1}\left(e^{-\frac{\theta}{2} \Pi_{1}}\right)$ as $\theta \rightarrow \infty$}

We shall now end the proof of Theorem 1.2 by showing :

Theorem 3.1 There is the equivalence result:

$$
E_{1}\left(e^{-\frac{\theta}{2} \Pi_{1}}\right) \underset{\theta \rightarrow \infty}{\sim} \frac{C}{\theta^{\frac{1}{3}}}
$$

\section{Proof of Theorem 3.1}

We recall that, from Theorem 1.3 :

$$
E_{1}\left(e^{-\frac{\theta}{2} \Pi_{1}}\right)=\frac{2 K_{i \nu}(\sqrt{4 \theta})}{K_{i \nu}(\sqrt{4 \theta})-\sqrt{4 \theta} K_{i \nu}^{\prime}(\sqrt{4 \theta})}
$$

with $\nu=\sqrt{4 \theta-1}\left(\theta \geq \frac{1}{4}\right)$. We shall successively find an equivalent of the numerator and the denominator of (3.2), the difficulty arising from the fact that, in $K_{i \nu}(\sqrt{4 \theta})$ (and 
$\left.K_{i \nu}^{\prime}(\sqrt{4 \theta})\right)$ the argument $\sqrt{4 \theta}$ and index $i \nu=i \sqrt{4 \theta-1}$ tend both to infinity as $\theta \rightarrow \infty$. To overcome this difficulty, we shall use some results about Bessel functions found in Watson ([Wat], p. 245-248), which we now recall.

i) Let $H_{i \nu}^{(1)}$ the first Hankel function (see [Leb], p. 120) ; it is related to $K_{i \nu}$ via the formula :

$$
K_{i \nu}(z)=\frac{i \pi}{2} e^{-\frac{\nu \pi}{2}} H_{i \nu}^{(1)}\left(z e^{\frac{i \pi}{2}}\right)
$$

We define $\epsilon$ by the formula : $i \nu=i z(1-\epsilon)$ and assume that, as $z \rightarrow \infty, \epsilon$ remains bounded (with, of course, $\nu$ depending on $z$ ). Then, there is the second order asymptotic expansion :

$$
H_{\nu}^{(1)}(z)=-\frac{2}{3 \pi}\left\{e^{\frac{2}{3} \pi i}\left(\sin \frac{\pi}{3}\right) \frac{\Gamma\left(\frac{1}{3}\right)}{\left(\frac{1}{6} z\right)^{\frac{1}{3}}}+e^{\frac{4}{3} \pi i}(\epsilon z)\left(\sin \frac{2 \pi}{3}\right) \frac{\Gamma\left(\frac{2}{3}\right)}{\left(\frac{1}{6} z\right)^{\frac{2}{3}}}+o\left(\frac{1}{z^{\frac{2}{3}}}\right)\right\}
$$

ii) Let us study the numerator of (3.2) :

$$
\begin{aligned}
& N= 2 K_{i \nu}(\sqrt{4 \theta})=2 \frac{i \pi}{2} e^{-\frac{\nu \pi}{2}} H_{i \nu}^{(1)}\left(e^{\frac{i \pi}{2}} \sqrt{4 \theta}\right) \\
&=(i \pi) e^{-\frac{\nu \pi}{2}}\left(-\frac{2}{3 \pi}\right)\left\{C_{1} e^{\frac{2}{3} \pi i} \frac{1}{\left(e^{\frac{i \pi}{2}} \sqrt{4 \theta}\right)^{\frac{1}{3}}}+o\left(\frac{1}{\theta^{\frac{1}{6}}}\right)\right\} \\
&\left(\text { with } C_{1}=\left(\sin \frac{\pi}{3}\right) \Gamma\left(\frac{1}{3}\right) 6^{\frac{1}{3}}\right) \\
& \underset{\theta \rightarrow \infty}{\sim} \frac{2^{\frac{2}{3}}}{3} C_{1} e^{-\frac{\nu \pi}{2}} \frac{1}{\theta^{\frac{1}{6}}}
\end{aligned}
$$

Here, we have used the first order expansion (3.4) with : $e^{\frac{2}{3} i \pi} \cdot e^{-\frac{i \pi}{6}}=i\left(\frac{4 \pi}{6}-\frac{\pi}{6}=\frac{3 \pi}{6}=\frac{\pi}{2}\right)$ and the fact that here

$$
\begin{aligned}
\epsilon z & =z-\nu \\
& =i \sqrt{4 \theta}-i \sqrt{4 \theta-1}=i \sqrt{4 \theta}\left(1-\sqrt{1-\frac{1}{4 \theta}}\right) \underset{\theta \rightarrow \infty}{\longrightarrow} 0
\end{aligned}
$$

iii) We now study the denominator of (3.2)

$$
\begin{aligned}
D & =K_{i \nu}(\sqrt{4 \theta})-\sqrt{4 \theta} K_{i \nu}^{\prime}(\sqrt{4 \theta}) \\
& =K_{i \nu}(\sqrt{4 \theta})+\sqrt{4 \theta} K_{i \nu-1}(\sqrt{4 \theta})+i \sqrt{4 \theta-1} K_{i \nu}(\sqrt{4 \theta})
\end{aligned}
$$

(after using $\frac{d}{d z}\left(z^{\mu} K_{\mu}(z)=-z^{\mu} K_{\mu-1}(z)\right)$; [Leb], p. 110). Since we have already studied the asymptotic behavior of $K_{i \nu}(\sqrt{4 \theta})$, it remains to study that of

$$
\Delta(\theta):=\sqrt{4 \theta} K_{i \nu-1}(\sqrt{4 \theta})+i \sqrt{4 \theta-1} K_{i \nu}(\sqrt{4 \theta})
$$


Now, since $\quad \sqrt{4 \theta} K_{i \nu-1}(\sqrt{4 \theta})+i \sqrt{4 \theta-1} K_{i \nu}(\sqrt{4 \theta})$ is real (from (2.18)) and since $i \sqrt{4 \theta-1} K_{i \nu}(\sqrt{4 \theta})$ is purely imaginary,

the development of $\Delta(\theta)$ as $\theta \rightarrow \infty$ is that of the real part of $\sqrt{4 \theta} K_{i \nu-1}(\sqrt{4 \theta})$. Then, we obtain :

$$
\begin{aligned}
\sqrt{4 \theta} K_{i \nu-1}(\sqrt{4 \theta}) & =\sqrt{4 \theta} \frac{i \pi}{2} e^{\frac{i \pi}{2}(i \nu-1)} H_{i \nu-1}^{(1)}\left(e^{\frac{i \pi}{2}} \sqrt{4 \theta}\right) \\
& =\sqrt{4 \theta} \frac{\pi}{2} e^{-\frac{\nu \pi}{2}} H_{i \nu-1}^{(1)}\left(e^{\frac{i \pi}{2}} \sqrt{4 \theta}\right)
\end{aligned}
$$

from (3.3), by replacing $i \nu$ by $i \nu-1$. This time, we use the second order development (3.4) with here : $z=e^{\frac{i \pi}{2}} \sqrt{4 \theta}$ and :

$$
\begin{aligned}
i \nu-1=z(1-\epsilon) & =i \sqrt{4 \theta}(1-\epsilon) \text {, i.e. : } \quad \epsilon z=\epsilon e^{\frac{i \pi}{2}} \sqrt{4 \theta} \\
\text { or } \quad \epsilon z & =\epsilon e^{\frac{i \pi}{2}} \sqrt{4 \theta}=1+\frac{1}{4 \sqrt{\theta}}+o\left(\frac{1}{\sqrt{\theta}}\right) \quad(\nu=\sqrt{4 \theta-1})
\end{aligned}
$$

We obtain :

$$
\begin{aligned}
& \sqrt{4 \theta} K_{i \nu-1}(\sqrt{4 \theta})=\sqrt{4 \theta} \frac{\pi}{2} e^{-\frac{\nu \pi}{2}}\left(-\frac{2}{3} \pi\right) \times \cdots \\
& \quad \cdots\left\{C_{1} e^{\frac{2 i \pi}{3}} \frac{1}{\left(e^{\frac{i \pi}{2}} \sqrt{4 \theta}\right)^{\frac{1}{3}}}+C_{2} e^{\frac{4 i \pi}{3}}\left(1+\frac{i}{4 \sqrt{\theta}}\right) \cdot \frac{1}{\left(e^{\frac{i \pi}{2}} \sqrt{4 \theta}\right)^{\frac{2}{3}}}+o\left(\frac{1}{\theta^{\frac{1}{3}}}\right)\right\}
\end{aligned}
$$

with $C_{1}=\left(\sin \frac{\pi}{3}\right) \Gamma\left(\frac{1}{3}\right) 6^{\frac{1}{3}}$ and $C_{2}=\left(\sin \frac{2 \pi}{3}\right) \Gamma\left(\frac{2}{3}\right) 6^{\frac{2}{3}} \quad\left(C_{1}, C_{2}>0\right)$

$$
=-\frac{1}{3} \sqrt{4 \theta} e^{-\frac{\nu \pi}{2}}\left\{C_{1} i \frac{1}{(\sqrt{4 \theta})^{\frac{1}{3}}}-C_{2}\left(1+\frac{i}{4 \sqrt{\theta}}\right) \frac{1}{\left(\sqrt{4 \theta}^{\frac{2}{3}}\right.}+o\left(\frac{1}{\theta^{\frac{1}{3}}}\right)\right\}
$$

Considering now the real part of (3.12) to obtain :

$$
\begin{aligned}
& \Delta(\theta)=\mathcal{R} e\left(\sqrt{4 \theta} K_{i \nu-1}(\sqrt{4 \theta})+i \sqrt{4 \theta-1} K_{i \nu}(\sqrt{4 \theta})\right) \\
&=\frac{1}{3} \sqrt{4 \theta} e^{-\frac{\nu \pi}{2}} C_{2}\left(\frac{1}{(\sqrt{4 \theta})^{\frac{2}{3}}}+o\left(\frac{1}{\theta^{\frac{1}{3}}}\right)\right) \\
& \underset{\theta \rightarrow \infty}{\sim} \frac{2^{\frac{1}{3}} C_{2}}{3} e^{-\frac{\nu \pi}{2}} \theta^{\frac{1}{6}}
\end{aligned}
$$

- We then gather (3.2), (3.6) and (3.13), we obtain :

$$
\begin{aligned}
E_{1}\left(e^{-\frac{\theta}{2} \Pi_{1}}\right) \underset{\theta \rightarrow \infty}{\sim} \frac{2 \frac{2^{\frac{2}{3}}}{3} C_{1} e^{-\frac{\nu \pi}{2}} \frac{1}{\theta^{\frac{1}{6}}}}{\frac{2^{\frac{2}{3}}}{3} C_{1} e^{-\frac{\nu \pi}{2}} \frac{1}{\theta^{\frac{1}{6}}}+\frac{2^{\frac{1}{3}}}{3} C_{2} e^{-\frac{\nu \pi}{2}} \theta^{\frac{1}{6}}} \\
\underset{\theta \rightarrow \infty}{\sim} \frac{2^{\frac{4}{3}} C_{1}}{C_{2}} \frac{1}{\theta^{\frac{1}{3}}}=\frac{2}{3^{\frac{1}{3}}} \frac{\Gamma\left(\frac{1}{3}\right)}{\Gamma\left(\frac{2}{3}\right)} \frac{1}{\theta^{\frac{1}{3}}}
\end{aligned}
$$


from the explicit formulae for $C_{1}$ and $C_{2}$. This is Theorem 3.1.

Remark 3.2 Using Theorem 1.3, we obtain, for $0<x \leq 1$ :

$$
E_{x}\left(e^{-\frac{\theta}{2} \Pi_{1}}\right)=1+x \frac{\frac{1}{2} K_{i \sqrt{4 \theta-1}}(\sqrt{4 \theta})+\sqrt{\theta} K_{i \sqrt{4 \theta-1}}^{\prime}(\sqrt{4 \theta})}{\frac{1}{2} K_{i \sqrt{4 \theta-1}}(\sqrt{4 \theta})-\sqrt{\theta} K_{i \sqrt{4 \theta-1}}^{\prime}(\sqrt{4 \theta})}
$$

It now follows easily from (3.6) and (3.13) that :

$$
E_{x}\left(e^{-\frac{\theta}{2} \Pi_{1}}\right) \underset{\theta \rightarrow \infty}{\longrightarrow} 1-x
$$

Now, on the other hand :

$$
E_{x}\left(e^{-\frac{\theta}{2} \Pi_{1}}\right) \underset{\theta \rightarrow \infty}{\longrightarrow} P_{x}\left(\Pi_{1}=0\right)
$$

We recall (and recover here) that $P_{x}\left(\Pi_{1}=0\right)=1-x$. This is point ii) of Theorem 1.2.

\section{Extending the preceding results to the variables $\Pi_{k}^{(\rho, x)}$ with}

$$
\Pi_{k}^{(\rho, x)}:=\int_{0}^{\infty}\left(e^{\rho\left(\log x+B_{t}-\frac{t}{2}\right)}-k\right)^{+} d t
$$

$(\rho, x, k>0)$. In the preceding Sections, we have studied the case $\rho=1$.

The analogue of Theorem 1.3 may be stated as :

Theorem 4.1 The Laplace transform of $\Pi_{1}^{(\rho)}$ under $P_{x}$ is given by :

$$
E_{x}\left(e ^ { - \frac { \theta } { 2 } \Pi _ { 1 } ^ { ( \rho ) } ) } \left\{\begin{array}{cl}
\frac{\sqrt{x} K_{\gamma}\left(\frac{\sqrt{4 \theta}}{\rho} x^{\frac{\rho}{2}}\right)}{\frac{1}{2} K_{\gamma}\left(\frac{\sqrt{4 \theta}}{\rho}\right)-\sqrt{\theta} K_{\gamma}^{\prime}\left(\frac{\sqrt{4 \theta}}{\rho}\right)} & \text { if } x \geq 1 \\
1+x \frac{\frac{1}{2} K_{\gamma}\left(\frac{\sqrt{4 \theta}}{\rho}\right)+\sqrt{\theta} K_{\gamma}^{\prime}\left(\frac{\sqrt{4 \theta}}{\rho}\right)}{\frac{1}{2} K_{\gamma}\left(\frac{\sqrt{4 \theta}}{\rho}\right)-\sqrt{\theta} K_{\gamma}^{\prime}\left(\frac{\sqrt{4 \theta}}{\rho}\right)} & \text { if } 0<x \leq 1
\end{array}\right.\right.
$$

with $\gamma=\frac{\sqrt{1-4 \theta}}{\rho}$ if $\theta \leq \frac{1}{4}$ and $\gamma=i \frac{\sqrt{4 \theta-1}}{\rho}$ if $\theta>\frac{1}{4}$.

The proof of this Theorem 4.1 is quite similar to that of Theorem 1.3. It hinges upon the following Lemma 4.2. 


\section{Lemma 4.2}

i) Let, for $\theta \geq 0$ and $\rho>0$, the function $\varphi_{\theta}^{(\rho)}: \mathbb{R}_{+} \rightarrow \mathbb{R}$ defined by :

$$
\varphi_{\theta}^{(\rho)}(y)=\sqrt{y} K_{\gamma}\left(\frac{\sqrt{4 \theta}}{\rho} y^{\frac{\rho}{2}}\right) \quad(y \geq 0)
$$

Then, it satisfies :

$$
\varphi_{\theta}^{(\rho)^{\prime \prime}}(y)+\theta\left(-y^{\rho-2}+\frac{1}{y^{2}}\right) \varphi_{\theta}^{(\rho)}(y)=0
$$

(see [Pet], p. 306, with $\rho-2=m$, formula (8), after taking care of replacing a by $-a)$.

ii) Let $\widetilde{\varphi}_{\theta}^{(\rho)}: \mathbb{R}_{+} \rightarrow \mathbb{R}$ defined by :

$$
\widetilde{\varphi}_{\theta}^{(\rho)}(y)= \begin{cases}\varphi_{\theta}^{(\rho)}(y) & \text { if } y \geq 1 \\ \left.\varphi_{\theta}^{(\rho)}(1)-\varphi_{\theta}^{(\rho)^{\prime}}(1)+y \varphi_{\theta}^{(\rho)}\right)^{\prime}(1) & \text { if } y \leq 1\end{cases}
$$

Then $\widetilde{\varphi}_{\theta}^{(\rho)}$ is positive, decreasing, convex and satisfies :

$$
\widetilde{\varphi}_{\theta}^{(\rho)}(y)+\theta\left(-y^{\rho-2}+\frac{1}{y^{2}}\right) 1_{y \geq 1} \widetilde{\varphi}_{\theta}^{(\rho)}(y)=0
$$

Remark 4.1 As a check, we note that formula (4.2) allows to recover the identity (1.8) :

$$
\int_{0}^{\infty} e^{\rho B_{t}-\frac{\rho t}{2}} d t \stackrel{(\text { law })}{=} \frac{2}{\rho^{2} \gamma_{\frac{1}{\rho}}}
$$

Indeed, on one hand :

$$
E\left(e^{-\frac{\theta}{2} \frac{2}{\rho^{2} \gamma \frac{1}{\rho}}}\right)=\frac{1}{\Gamma\left(\frac{1}{\rho}\right)} \int_{0}^{\infty} e^{-\frac{\theta}{\rho^{2} z}-z} z^{\frac{1}{\rho}-1} d z=\frac{2}{\Gamma\left(\frac{1}{\rho}\right)} K_{\frac{1}{\rho}}\left(\frac{\sqrt{4 \theta}}{\rho}\right)\left(\frac{\theta}{\rho^{2}}\right)^{\frac{1}{2 \rho}} \text { (see [Leb], p. }
$$

On the other hand :

$$
\begin{aligned}
E_{1}\left(e^{-\frac{\theta}{2} \Pi_{0}^{(\rho)}}\right) & =\lim _{\epsilon \downarrow 0} E_{1}\left(\exp -\frac{\theta}{2} \int_{0}^{\infty}\left(e^{\rho B_{t}-\frac{\rho t}{2}}-\varepsilon\right)^{+} d t\right) \\
& =\lim _{\epsilon \downarrow 0} E_{1}\left(\exp -\frac{\theta \varepsilon}{2} \int_{0}^{\infty}\left(e^{\rho\left(\frac{\log \frac{1}{\varepsilon}}{\rho}+B_{t}\right)-\frac{\rho t}{2}}-1\right)^{+} d t\right) \\
& =\lim _{\epsilon \downarrow 0} \frac{\left(\frac{1}{\varepsilon}\right)^{\frac{1}{2 \rho}} K_{\frac{\sqrt{1-4 \theta \varepsilon}}{\rho}}\left(\frac{\sqrt{4 \theta \varepsilon}}{\rho}\right)\left(\frac{1}{\varepsilon}\right)^{\frac{1}{\rho} \frac{\rho}{2}}}{\frac{1}{2} K_{\frac{\sqrt{1-4 \theta \varepsilon}}{\rho}}\left(\frac{\sqrt{4 \theta \varepsilon}}{\rho}\right)-\sqrt{\theta \varepsilon} K_{\frac{\sqrt{1-4 \theta \varepsilon}}{\rho}}^{\prime}\left(\frac{\sqrt{4 \theta \varepsilon}}{\rho}\right)}
\end{aligned}
$$


from (4.2), replacing $\theta$ by $\theta \varepsilon$ and $x$ by $\left(\frac{1}{\varepsilon}\right)^{\frac{1}{\rho}}$

$$
\begin{aligned}
& \underset{\varepsilon \rightarrow 0}{\sim} \frac{\left(\frac{1}{\varepsilon}\right)^{\frac{1}{2 \rho}} K_{\frac{1}{\rho}}\left(\frac{\sqrt{4 \theta}}{\rho}\right)}{\frac{1}{2} K_{\frac{\sqrt{1-4 \theta \varepsilon}}{\rho}}\left(\frac{\sqrt{4 \theta \varepsilon}}{\rho}\right)-\frac{\rho}{2}\left(\frac{\sqrt{4 \theta \varepsilon}}{\rho}\right) K_{\frac{\sqrt{1-4 \theta \varepsilon}}{\rho}}^{\prime}\left(\frac{\sqrt{4 \theta \varepsilon}}{\rho}\right)} \\
& \left.\underset{\varepsilon \rightarrow 0}{\sim} \frac{\left(\frac{1}{\varepsilon}\right)^{\frac{1}{2 \rho}} K_{\frac{1}{\rho}}\left(\frac{\sqrt{4 \theta}}{\rho}\right)}{\frac{\rho}{2}\left[\frac{\sqrt{4 \theta \varepsilon}}{\rho} K_{\frac{1}{\rho}+1}\left(\frac{\sqrt{4 \theta \varepsilon}}{\rho}\right)\right.} \quad \text { (since } z K^{\prime} \nu=\nu K_{\nu}(z)-z K_{\nu+1}(z)\right) \\
& \underset{\varepsilon \rightarrow 0}{\sim} \frac{\left(\frac{1}{\varepsilon}\right)^{\frac{1}{2 \rho}} K_{\frac{1}{\rho}}\left(\frac{\sqrt{4 \theta}}{\rho}\right)}{\frac{\rho}{2}\left(\Gamma\left(\frac{1}{\rho}+1\right)\left(\frac{\sqrt{4 \theta \varepsilon}}{\rho}\right)^{-\frac{1}{\rho}}\right.} \quad\left(\text { since } K_{\nu}(z) \sim \frac{1}{2} \Gamma(\nu)\left(\frac{z}{2}\right)^{-1}\right) \\
& =\frac{\left(\frac{1}{\varepsilon}\right)^{\frac{1}{2 \rho}} K_{\frac{1}{\rho}}\left(\frac{\sqrt{4 \theta}}{\rho}\right)}{\Gamma\left(\frac{1}{\rho}\right)} \frac{\varepsilon^{\frac{1}{2 \rho}} \theta^{\frac{1}{2 \rho}}}{\rho^{\frac{1}{\rho}}} \\
& \underset{\varepsilon \downarrow 0}{\longrightarrow}\left(\frac{\theta}{\rho^{2}}\right)^{\frac{1}{2 \rho}} \frac{2}{\Gamma\left(\frac{1}{\rho}\right)} K_{\frac{1}{\rho}}\left(\frac{\sqrt{4 \theta}}{\rho}\right)=E\left(e^{-\frac{\theta}{2} \Pi_{0}^{(\rho)}}\right)
\end{aligned}
$$

\section{Remark 4.2}

Taking up again the arguments of the proof of Theorem 3.1, it is not difficult to see that :

$$
E_{1}\left(e^{-\frac{\theta}{2} \Pi_{1}^{(\rho)}}\right) \underset{\theta \rightarrow \infty}{\sim} \frac{C(\rho)}{\theta^{\frac{1}{3}}}
$$

where $C(\rho)$ is a strictly positive constant, depending on $\rho$. We then deduce that, for $\alpha<0$ :

$$
E_{1}\left(\left(\Pi_{1}^{(\rho)}\right)^{\alpha}\right)<\infty \text { if and only if }|\alpha|<\frac{1}{3}
$$

On the other hand, it is not difficult to see that :

- If $x>1$, for all $\alpha<0, E_{1}\left(\left(\Pi_{1}^{(\rho)}\right)^{\alpha}\right)<\infty$.

- If $x<1$, for all $\alpha<0, E_{1}\left(\left(\Pi_{1}^{(\rho)}\right)^{\alpha}\right)=+\infty$.

Concerning the positive moments of $\Pi_{1}^{(\rho)}$ :

$$
\text { - If } \quad 0<\alpha<\frac{1}{\rho}, \text { then } E_{x}\left(\left(\Pi_{1}^{(\rho)}\right)^{\alpha}\right)<\infty
$$

Indeed (4.7), for $x=1$, follows from :

$$
\begin{aligned}
E_{1}\left(\left(\Pi_{1}^{(\rho)}\right)^{\alpha}\right) & \leq E_{1}\left(\left(\Pi_{0}^{(\rho)}\right)^{\alpha}\right)=\frac{1}{\Gamma\left(\frac{1}{\rho}\right)} \int_{0}^{\infty}\left(\frac{2}{\rho^{2} z}\right)^{\alpha} e^{-z} z^{\frac{1}{\rho}-1} d z \\
& <\infty \text { if } \alpha<\frac{1}{\rho} \\
(\text { since } & \left.\Pi_{0} \stackrel{\text { (law) }}{=} \frac{2}{\rho^{2} \gamma_{\frac{1}{\rho}}}, \text { from }(1.10)\right)
\end{aligned}
$$


The fact that $E_{x}\left(\left(\Pi_{1}^{(\rho)}\right)^{\alpha}\right)<\infty$ for every $x>0$, and every $\alpha<\frac{1}{\rho}$ may be obtained by using arguments close to those used in the proof of Theorem 1.1.

We believe that, for all $\rho>0$ :

$$
E_{x}\left(\left(\Pi_{1}^{(\rho)}\right)^{\frac{1}{\rho}}\right)=+\infty .
$$

We now show (4.8), when $\rho>1$

It then suffices, by using the arguments of the proof of Theorem 1.1, to see that (4.8) is true when $x=1$.

- We first show that : $1-E_{1}\left(e^{-\frac{\theta}{2} \Pi_{1}^{(\rho)}}\right) \underset{\theta \rightarrow 0}{\sim} C \theta^{\frac{1}{\rho}}$

Indeed, from Theorem 4.1, we have :

$$
E_{1}\left(\exp -\frac{\theta}{2} \Pi_{1}^{(\rho)}\right)=1+\frac{\frac{1}{2} K_{\gamma}\left(\frac{\sqrt{4 \theta}}{\rho}\right)+\sqrt{\theta} K_{\gamma}^{\prime}\left(\frac{\sqrt{4 \theta}}{\rho}\right)}{\frac{1}{2} K_{\gamma}\left(\frac{\sqrt{4 \theta}}{\rho}\right)-\sqrt{\theta} K_{\gamma}^{\prime}\left(\frac{\sqrt{4 \theta}}{\rho}\right)}
$$

with $\gamma=\frac{\sqrt{1-4 \theta}}{\rho}\left(\right.$ and $\left.\theta \leq \frac{1}{4}\right)$. Thus :

$$
\begin{aligned}
& 1- E_{1}\left(e^{-\frac{\theta}{2} \Pi_{1}^{(\rho)}}\right)=-\frac{\frac{1}{2} K_{\gamma}\left(\frac{\sqrt{4 \theta}}{\rho}\right)+\frac{\rho}{2}\left\{\frac{\sqrt{4 \theta}}{\rho} K_{\gamma}^{\prime}\left(\frac{\sqrt{4 \theta}}{\rho}\right)\right\}}{\frac{1}{2} K_{\gamma}\left(\frac{\sqrt{4 \theta}}{\rho}\right)-\frac{\rho}{2}\left\{\frac{\sqrt{4 \theta}}{\rho} K_{\gamma}^{\prime}\left(\frac{\sqrt{4 \theta}}{\rho}\right)\right\}} \\
&=-\frac{\frac{1}{2} K_{\frac{\sqrt{1-4 \theta}}{\rho}}\left(\frac{\sqrt{4 \theta}}{\rho}\right)\left(1-\frac{\sqrt{1-4 \theta}}{\rho}\right)-\frac{\sqrt{\theta}}{\rho} K_{\frac{\sqrt{1-4 \theta}}{\rho}-1}\left(\frac{\sqrt{4 \theta}}{\rho}\right)}{\frac{1}{2} K_{\frac{\sqrt{1-4 \theta}}{\rho}}\left(\frac{\sqrt{4 \theta}}{\rho}\right)\left(1+\frac{\sqrt{1-4 \theta}}{\rho}\right)+\frac{\sqrt{\theta}}{\rho} K_{\frac{\sqrt{1-4 \theta}}{\rho}-1}\left(\frac{\sqrt{4 \theta}}{\rho}\right)}
\end{aligned}
$$

(after using $\left.z K_{\mu}^{\prime}(z)=-\mu K_{\mu}(z)-z K_{\mu-1}(z)\right)$.

Since $\rho>1$, we replace $K_{\frac{\sqrt{1-4 \theta}}{\rho}-1}$ by $K_{1-\frac{\sqrt{1-4 \theta}}{\rho}}$ (since $K_{\mu}=K_{-\mu}$ ) and we deduce, from :

$$
K_{1-\frac{\sqrt{1-4 \theta}}{\rho}} \underset{\theta \rightarrow 0}{\longrightarrow} K_{1-\frac{1}{\rho}}, \quad \text { with } \quad 1-\frac{1}{\rho}>0 \text { : }
$$

that :

$$
\begin{aligned}
& 1-E_{1}\left(e^{-\frac{\theta}{2} \Pi_{1}^{(\rho)}}\right) \underset{\theta \rightarrow 0}{\sim} \frac{a \theta^{1-\frac{1}{2 \rho}}+b \theta^{\frac{1}{2 \rho}}}{a^{\prime} \theta^{-\frac{1}{2 \rho}}+b^{\prime} \theta^{\frac{1}{2 \rho}}}\left(\text { since, for } \mu>0 K_{\mu}(z) \underset{z \rightarrow 0}{\sim} C_{\mu} z^{-\mu} ;\right. \text { see [Leb], p. 136) } \\
& \underset{\theta \rightarrow 0}{\sim} \frac{b}{a^{\prime}} \theta^{\frac{1}{\rho}} \quad\left(\text { since } \rho \geq 1 \text { implies } 1-\frac{1}{2 \rho} \geq \frac{1}{2 \rho}\right)
\end{aligned}
$$

- From (4.9) we deduce :

$$
\begin{aligned}
\int_{0}^{\infty} e^{-\frac{\theta t}{2}} P\left(\Pi_{1}^{(\rho)} \geq t\right) d t & =\frac{2}{\theta}\left(1-E\left(e^{-\frac{\theta}{2} \Pi_{1}^{(\rho)}}\right)\right) \\
& \underset{\theta \rightarrow \infty}{\sim} C^{\prime} \theta^{\frac{1}{\rho}-1}
\end{aligned}
$$


Hence, from the Tauberian Theorem :

$$
P\left(\Pi_{1}^{(\rho)} \geq t\right) \underset{t \rightarrow \infty}{\sim} C^{\prime \prime} \frac{1}{t^{\frac{1}{\rho}}}
$$

and

$$
\begin{aligned}
E\left(\left(\Pi_{1}^{(\rho)}\right)^{\frac{1}{\rho}}\right) & =\int_{0}^{\infty} P\left(\left(\Pi_{1}^{(\rho)}\right)^{\frac{1}{\rho}} \geq t\right) d t \\
& =\int_{0}^{\infty} P\left(\Pi_{1}^{(\rho)} \geq t^{\rho}\right) d t=+\infty, \quad \text { from }(4.10) .
\end{aligned}
$$

We note that, for $\rho<1$, the preceding argument cannot be applied since :

$$
\int_{1}^{\infty} e^{-\frac{\theta t}{2}} \frac{d t}{t^{\frac{1}{\rho}}} \underset{\theta \rightarrow 0}{\longrightarrow} \int_{1}^{\infty} \frac{d t}{t^{\frac{1}{\rho}}}<\infty
$$

\section{References}

[Duf] D. Dufresne The distribution of a perpetuity, with applications to risk theory and pension funding. Scand. Actuarial J., p. 39-79, (1990).

[Kam] E. Kamke Differentialgleichungen. Lösungsmethoden and Lösungen. Becker-Erler Verlag, Leipzig, (1942).

[Leb] N.N. Lebedev Special functions and their applications. Dover Publ. (1972).

[MRY1] D. Madan, B. Roynette, M. Yor An alternative expression for the Black-Scholes formula in terms of Brownian first and last passage times. Prépublication IECN, $\mathrm{n}^{\circ}$ 08, (2008).

[MRY2] D. Madan, B. Roynette, M. Yor Unifying Black-Scholes type formulae which involve Brownian last passage times up to a finite horizon. Prépublication IECN, $\mathrm{n}^{\circ} 23$, (2008). To appear in Asia Pacific Markets Springer.

[MRY3] D. Madan, B. Roynette, M. Yor Put option prices as joint distribution functions in strike and maturity : the Black-Scholes case. Prépublication IECN, $n^{\circ} 41,(2008)$.

[NY] A. Nikeghbali, M. Yor Doob's maximal identity, multiplicative decomposition and enlargment of filtration. Illinois J. Maths Special Vol. for J. Doob, p. 791-814, (2005).

[Pet] G. Petiau La théorie des fonctions de Bessel. CNRS, (1955).

[RY] D. Revuz, M. Yor Continuous Martingales and Brownian motion. Third edition, Springer, Corrected $3^{\text {rd }}$ printing (2005). 
[SY1] P. Salminen, M. Yor Properties of perpetual integral functionals of Brownian motion with drift. Ann. IHP, 41 (3), p. 335-347, (2005).

[SY2] P. Salminen, M. Yor Perpetual integral functionals as hitting and occupation times. Elect. J. Prob., 10, p. 371-419, (2005).

[SY3] P. Salminen, M. Yor On Dufresne's perpetuities, translated and reflected. Stoch. proc. and applications to Math. finance, in Proceedings of the $3^{\text {rd }}$ International Symposium Ritsumeikan Univ. World Scientific Publishing Co, Inc., River Edge, p. 337-354, (2004).

[Wat] G.N. Watson A treatise on the theory of Bessel functions. Cambridge University Press, Cambridge, England. The Macmillan Company, New-York, (1944).

[Y] M. Yor On certain exponential functionals of real-valued Brownian motion. Paper 1, in Exp. functionals of Brownian motion and Related processes, Springer-Finance (2001). 